\title{
Ultrasound Image under Artificial Intelligence Algorithm in Thoracoscopic Surgery for Papillary Thyroid Carcinoma
}

\author{
Xin Shen $\mathbb{D}^{1},{ }^{1}$ Aolin Yuan $(\mathbb{D})^{2}$ and Kaili Zhang $\mathbb{D}^{1}$ \\ ${ }^{1}$ Breast AndThyroid Surgical Oncology, Hebei Medical University Second Affiliated Hospital, Shijiazhuang 050000, Hebei, China \\ ${ }^{2}$ Minimally Invasive Spine Surgery, Chengde Medical University Affiliated Hospital, Chengde, 067000, Hebei, China \\ Correspondence should be addressed to Kaili Zhang; 1420110217@st.usst.edu.cn
}

Received 26 October 2021; Revised 11 January 2022; Accepted 13 January 2022; Published 14 February 2022

Academic Editor: M Pallikonda Rajasekaran

Copyright (c) 2022 Xin Shen et al. This is an open access article distributed under the Creative Commons Attribution License, which permits unrestricted use, distribution, and reproduction in any medium, provided the original work is properly cited.

This study aimed to investigate the therapeutic effect of thoracoscopic thyroidectomy guided by an ultrasound image based on an artificial intelligence algorithm on papillary thyroid carcinoma. Patients diagnosed with papillary thyroid carcinoma by imaging examination or needle biopsy in hospital were selected. The subjects were randomly divided into the experimental group and the control group. In the experimental group, 94 patients underwent conventional thoracoscopic thyroid tumor resection. In the control group, 119 patients underwent thoracoscopic thyroidectomy under ultrasonic guidance based on an intelligent algorithm. The adoption effect of ultrasound imaging based on the least variance algorithm in thoracoscopic papillary thyroid carcinoma surgery was evaluated by comparing the differences between the two groups of patients after laparoscopic thyroidectomy. The results showed that the ultrasonic imaging resolution and sound-absorbing spot imaging effect based on an artificial intelligence algorithm were superior to those of conventional ultrasonic imaging. Compared with the control group, the average duration of surgery in the experimental group was reduced and the intraoperative blood loss, postoperative cumulative drainage volume, postoperative drainage tube duration, hospital stay, and pain evaluation were dramatically better $(P<0.05)$. It was confirmed that ultrasound-guided endoscopic thyroid surgery based on the least variance algorithm had better results than conventional surgery and showed the same safety as traditional surgery, which was of clinical promotion value. However, some evaluations were influenced by subjective judgment, and the accuracy of data conclusions needed to be further studied.

\section{Introduction}

Nodular thyroid disease (NTD) is a common type of thyroid disorder. Under high-resolution ultrasound, thyroid nodules are diagnosed in about $19 \% \sim 67 \%$ of the population, with a high prevalence in women and elderly patients [1]. Thyroid cancer is a common endocrine malignant tumor, and its prevalence is increasing in recent years. Thyroid cancer occurs in thyroid follicular cells and parathyroid cells. The common types are follicular carcinoma, undifferentiated carcinoma, papillary thyroid carcinoma, and medullary carcinoma of thyroid. Among them, the prevalence of papillary thyroid carcinoma is as high as over 90\% [2].

At present, the clinical treatment of papillary thyroid carcinoma mainly includes conservative treatment and surgical treatment. If the effect of conservative treatment is not ideal, surgical treatment is generally recommended [3]. Although the initial neck open goiter resection can effectively remove the lesion, the postoperative incision is obvious. In recent years, with the continuous development of ultrasound technology and thoracoscopic technology, thoracoscopic surgery based on ultrasound guidance can effectively reduce the incision and postoperative scar. It is highly respected by more and more doctors and more easily accepted by patients [4]. Compared with computed tomography (CT) and magnetic resonance imaging (MRI), the ultrasonic probe can not only be moved by hand but also cost less and has a better stability. It can present real-time tissue imaging with no obvious side effects to the patient or the operator. Despite these advantages, ultrasound imaging technology is still subject to many limitations in clinical application [5]. The traditional ultrasonic imaging 
technology refers to the interaction between the ultrasonic wave emitted by the ultrasonic probe and the measured object. The echo beam signals reflected from the surface and internal depressions of the measured object are processed together. Then, the external structure or internal structure size of the measured object is displayed through image processing technology [6].

At present, the delay-and-sum (DAS) algorithm is the most widely used artificial intelligence algorithm in ultrasonic signal processing. DAS algorithm delays and weights the signals received by each array cell and then obtains the maximum output. However, in practice, the accuracy of ultrasonic echo signal may be interfered by the complexity of the detection object and the diversity of the detection environment. Therefore, the traditional DAS technology has the disadvantages of poor image resolution and contrast [7]. To overcome this shortcoming, some researchers proposed an adaptive beamforming algorithm. Compared with the DAS algorithm, the adaptive algorithm uses the received echo data to dynamically calculate the weighting coefficient, and each point collected in the image can get a set of weighting vectors. The standard Capon algorithm is the earliest adaptive weighting algorithm, also known as the minimum variance (MV) imaging algorithm [8].

In this study, the efficacy of the MV beamforming algorithm in ultrasound imaging for thoracoscopic surgical treatment of papillary thyroid carcinoma was analyzed based on the adaptive beamforming algorithm under artificial intelligence.

\section{Research Methods}

2.1. Research Objects. Patients diagnosed with papillary thyroid carcinoma by imaging examination or needle biopsy in the hospital were selected. The subjects were randomly divided into the experimental group and the control group. In the experimental group, 94 patients underwent conventional thoracoscopic thyroid tumor resection. In the control group, S-NERVE portable ultrasound was adopted. 119 patients underwent thoracoscopic thyroidectomy under ultrasonic guidance based on an intelligent algorithm. This study had been approved by the Ethics Committee of Hospital, and all patients had signed the informed consent.

Inclusion criteria were as follows: (i) female patients, aged less than 45 years; (ii) patients with the diameter of the mass less than $1 \mathrm{~cm}$; (iii) those with no extracapsular thyroid invasion occurrence; (iv) patients in whom no obvious lymph node metastasis was found; (v) patients whose enlarged lymph nodes were not fused or fixed. Exclusion criteria were as follows: (i) patients who had no postoperative cosmetic requirements; (ii) patients whose distant metastasis of tumor was diagnosed before surgery; (iii) patients with recurrent papillary thyroid carcinoma. The following patients were not allowed to participate in the study: (i) those who had a previous history of neck surgery or radiotherapy; (ii) patients whose lymph node metastasis in the lateral region was confirmed by diagnosis; (iii) patients whose imaging examination suggested external thyroid infiltration; (iv) obese patients; (v) male patients.
Therefore, ultrasound imaging based on the MV algorithm was applied to perform thoracoscopic thyroid tumor resection under its guidance, and various intraoperative and postoperative indicators were observed. Compared with patients undergoing conventional thoracoscopic thyroid tumor resection, the therapeutic effect of thoracoscopic surgery guided by ultrasound imaging based on the MV algorithm for thyroid papillary carcinoma was explored, providing data support for its clinical application.

2.2. Observation Indexes. The main indexes to be observed in this study include the following: I. general clinical information of all subjects, such as age, BMI, and tumor lesion size; II. perioperative short-term efficacy indexes, such as the number of dissected lymph nodes, operative time, postoperative drainage duration, intraoperative blood loss, the total length of hospital stays, and total postoperative drainage volume; III. postoperative complications, such as temporary or permanent recurrent laryngeal nerve injury and hypocalcemia, incision infection, postoperative bleeding, chest wall and neck skin stiffness, or other discomforts; IV. postoperative subjective feelings of patients, including whether patients had neck pain, neck discomfort, and psychological acceptance of patients; V. the degree of postoperative neck or chest pain; VI. patients' satisfaction with postoperative cosmetic effects of surgical wounds; VII. whether the tumor recurred or metastasized during followup.

2.3. Minimum Variance Algorithm. The adaptive beamforming algorithm refers to dynamically calculating the weighting vector through the ultrasonic echo signal and weighting the echo data after the delay of different array elements. After that, the beams are assembled and processed to obtain the ultrasonic beam synthesis signal [7]. Its schematic diagram is shown in Figure 1.

Compared with the previous DAS technology, the adaptive beamforming technology adds a dynamic weighting module [9] to reduce the noise energy in the output signal. The output echo signal is represented by $C_{A D S}(o)$. The specific implementation steps of the MV algorithm are as follows. It is assumed that there is a receiving array of $N$ array elements to delay the reception of the echo data in the target area and combine it with adaptive weighting processing. The obtained beam synthesis output signal expression is shown in

$$
y(k)=w^{H}(k) x_{d}(k)=\sum_{i=1}^{N} w_{i}(k) x_{i}\left(k-\Delta_{i}\right),
$$

where $k$ represents the $k$-th sampling point, $x_{d}(k)=\left[x_{1} k-\Delta_{i}, \ldots, x_{n} k-\Delta_{d}\right]^{T}$ is the ultrasonic echo data after focusing delay, $\Delta_{i}$ indicates the time to increase the delay of each element signal, $w(k)=\left[w_{1} k, \ldots, w_{n}(k)\right]^{T}$ represents the adaptive weighting coefficient, and $[\cdot]^{T}$ is the transposition operation. 


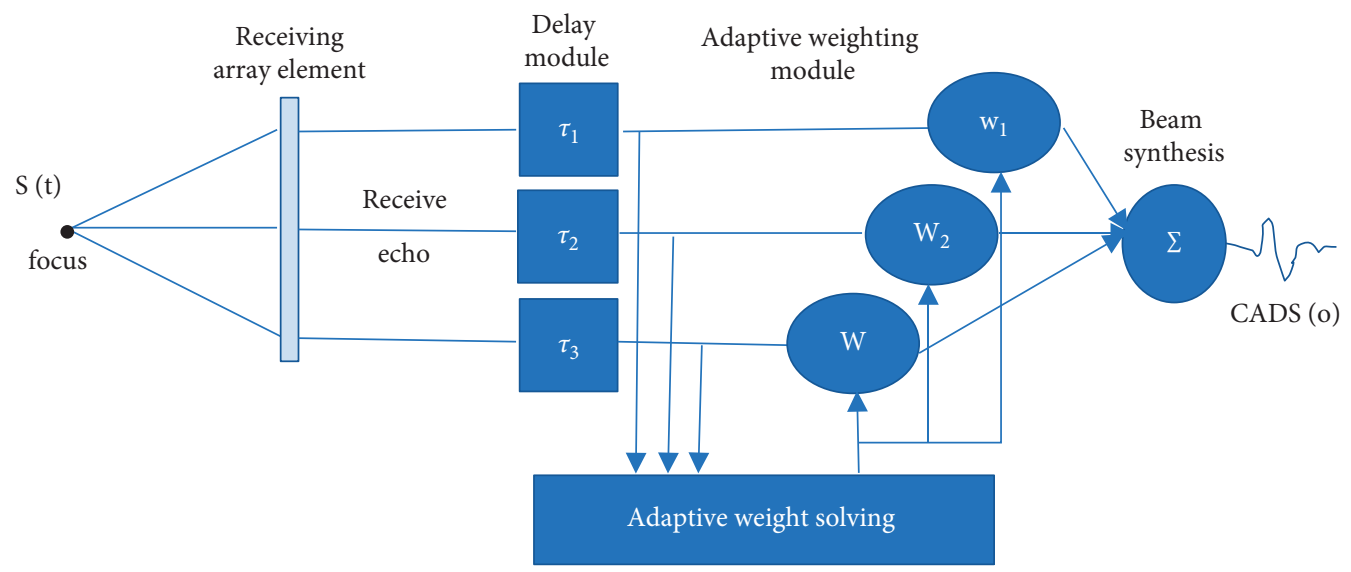

FIgUre 1: Adaptive beam synthesis.

According to the principle of minimum variance, the problem of solving weight vector $\mathrm{W}$ by $\mathrm{MV}$ algorithm is expressed as

$$
{ }_{w}^{\min } m^{H} R m \text {, subject to } m^{H} a=1 .
$$

$R=E\left[x_{d} x_{d}^{H}\right]$ is the sample covariance matrix of $N \times N$ dimensions, and $a=[1,1 \ldots, 1] T$ is the direction vector of $N \times 1$ dimensions. According to the Lagrange number multiplication equation, the optimal weight vector obtained is shown in

$$
w_{o p t}=\frac{R^{-1} a}{a^{H} R^{-1} a} .
$$

Finally, the output expression of the minimum variance algorithm is shown in

$$
y_{m v}(k)=w_{o p t}^{H} x_{d}(k) .
$$

2.4. Image Evaluation. The commonly used ultrasound imaging evaluation indexes mainly include the evaluation of the spatial resolution of point target imaging and evaluation of the contrast of sound absorption spot imaging [10]. Due to the complicated research on algorithm complexity in this research, here is a brief description of some operations:

(i) The Spatial Resolution of Point Target Imaging. The application of spatial resolution for point target imaging is used to evaluate the imaging effect. The imaging target point can only be used to measure the focus accuracy of the imaging algorithm through the position but not the size. Generally, the smaller and brighter the target point in the image is, the better the resolution of the algorithm and the more accurate the focus. The spatial resolution mainly includes axial resolution and horizontal resolution, which represent vertical and horizontal artifacts, respectively [11]. Axial resolution is the shortest distance between two distinguishable target points on the axis of the sound beam, expressed in $r_{c}$.

$$
r_{c}=e \frac{\tau_{q}}{2}=\frac{e}{2 B_{m}} .
$$

Here, $e$ is the speed of sound, $\tau_{q}$ is the pulse effective time, and $B_{m}$ is the pulse bandwidth. If it is necessary to improve the axial resolution of ultrasound imaging, the pulse wavelength or pulse period should be shortened, which will cause the reduction of ultrasound in the object to be measured and reduce the depth of detection. The lateral resolution refers to the shortest distance between two target points that can be distinguished in the direction perpendicular to the axis of the sound beam in the imaging area, which is shown in

$$
r_{1}=2 F \sin \frac{\theta_{m l}}{2}
$$

$F$ is the distance between the center of the array element and the target point, and $\theta_{m l}$ represents the angle between the two target points and the center of the array element. The lateral resolution is proportional to the effective width of the ultrasonic beam, and the lateral resolution is usually expressed by the width of the main lobe of $-6 \mathrm{~dB}$ or $-20 \mathrm{~dB}$. In addition, point target imaging usually indicates the amount of imaging artifacts by the amplitude of the first side lobe, and the amplitude of the side lobe is also proportional to the imaging artifacts.

(ii) Evaluation of Sound Absorption Spot Imaging. It is evaluated by contrast and background area variance. The specific analysis is as follows:

The difference between the brightness of the brightest and darkest areas in an image is the contrast ratio (CR), which is proportional to the imaging effect [12], whose mathematical definition is shown in

$$
C R=20 * 1_{g}\left(\frac{\mu_{A}}{\mu_{B}}\right) .
$$


Here, $\mu_{A}$ and $\mu_{B}$ are the mean values of the echo signals in the background area and the target area, respectively.

In real ultrasound imaging applications, the value of contrast is simplified as the difference between the mean value of the echo signal in the background area and the mean value of the echo signal in the target area. The brighter the imaging effect, the stronger the signal strength, and the higher the mean value of the corresponding echo signal.

In ultrasound imaging, the background area variance (standard deviation) is usually used to represent the robustness of the algorithm. The smaller the value, the better the robustness of the algorithm. The mathematical expression of background regional variance is shown in

$$
S D=\sqrt{\sigma_{A}^{2}+\sigma_{B}^{2}} .
$$

$\sigma_{A}$ represents the background area of the sound absorption spot, and $\sigma_{B}$ represents the signal standard deviation of the target area. This parameter is solved by directly applying the intensity standard deviation of the echo signal in the background area in the actual simulation imaging. In sound absorption spot imaging, the larger the variance between CR and the background area, the worse the imaging effect of the algorithm.

2.5. Statistical Methods. Data were processed by SPSS 22.0 data statistical software. The measurement data were expressed as mean \pm standard deviation, and the two groups of data were compared using the $t$-test of two independent samples. The difference of comparison rate was tested by $\chi^{2}$ four-grid table, and $P<0.05$ was considered statistically remarkable between the two groups.

\section{Research Results}

3.1. Evaluation of Ultrasound Imaging. Compared with the conventional traditional DAS algorithm, the point target imaging resolution of the MV algorithm was better, and the main lobe width and side-lobe peak display were dramatically reduced (Figure 2).

Contrast ratio (CR) is defined as the difference between the external average power and the average power of the central dark spot. The larger the value, the greater the degree of discrimination of the imaging area. The smaller the average power value, the worse the signal strength, and the darker the corresponding image area. The background area variance is a parameter that reflects the robustness of the algorithm. The smaller the value, the better the robustness of the algorithm [13]. The results are shown in Figure 3.

3.2. Basic Information of the Two Groups of Patients. According to the above inclusion and exclusion criteria, a total of 213 patients were included in this experiment, including 94 in the control group and 119 in the experimental group. The age of the experimental group was mainly distributed between 14 and 45 years old, and the average age was $(34.33 \pm 7.34)$ years old. The age of the control group was mainly distributed between 21 and 46 years old, and the average age was $(35.34 \pm 7.67)$ years old. The difference in age distribution between the experimental group and the control group was not statistically remarkable $(P>0.05)$. The average BMI index of the experimental group was $(21.02 \pm 1.23) \mathrm{kg} / \mathrm{m}^{2}$, the average BMI index of the control group was $(21.53 \pm 1.25) \mathrm{kg} / \mathrm{m}^{2}$, and the BMI index of the two groups was not statistically remarkable $(P>0.05)$ (Table 1).

3.3. Relevant Indexes after Surgery. According to the postoperative routine pathological results, there was no considerable difference in tumor size between the experimental group and the control group $(P>0.05)$. Compared with the experimental group, the operation duration of the control group was longer, and there was a considerable difference in the operation duration between the two groups, which was statistically remarkable $(P<0.05)$. The intraoperative blood loss in the control group was less than that in the experimental group, and the difference in blood loss between the two groups was statistically remarkable $(P<0.05)$. The cumulative drainage volume of the control group was more than that of the experimental group, and the difference between the two groups was substantial $(P<0.05)$. The drainage tube was placed for more days in the control group than in the experimental group, and the difference was substantial $(P<0.05)$. The number of days of hospitalization in the control group was more than that in the experimental group, with substantial differences $(P<0.05)$. There was little difference in the numbers between the two groups, and there was no statistical significance $(P>0.05)$ (Figure 4$)$.

3.4. Comparison of Postoperative Complications between the Experimental Group and the Control Group. A total of 19 patients in the control group had postoperative complications, accounting for $20.21 \%$, and a total of 14 patients in the experimental group had postoperative complications, accounting for $11.76 \%$. Compared with the control group, the experimental group had a slightly higher proportion of complications, but there was no considerable difference in the probability of complications between the two groups. Moreover, no fatal complications were found in all subjects after surgery (Table 2).

3.5. Comparison of Patients' Subjective Feelings after Surgery. The postoperative neck discomfort, neck pain, psychological acceptance, and satisfaction with postoperative incision scars of the experimental group were better than those of the control group. The differences were considerable $\left(\chi^{2}=7.032\right.$, 7.358, 19.013, $P<0.05$ ) (Figure 5).

3.6. Analysis of the Results of Postoperative Neck and Anterior Chest Wall Pain in the Experimental Group and the Control Group. According to the "facial expression pain scale", the degree of pain in the neck or anterior chest wall after the operation was evaluated (Figure 6). The score of pain was positively correlated. When the pain had a serious impact on 


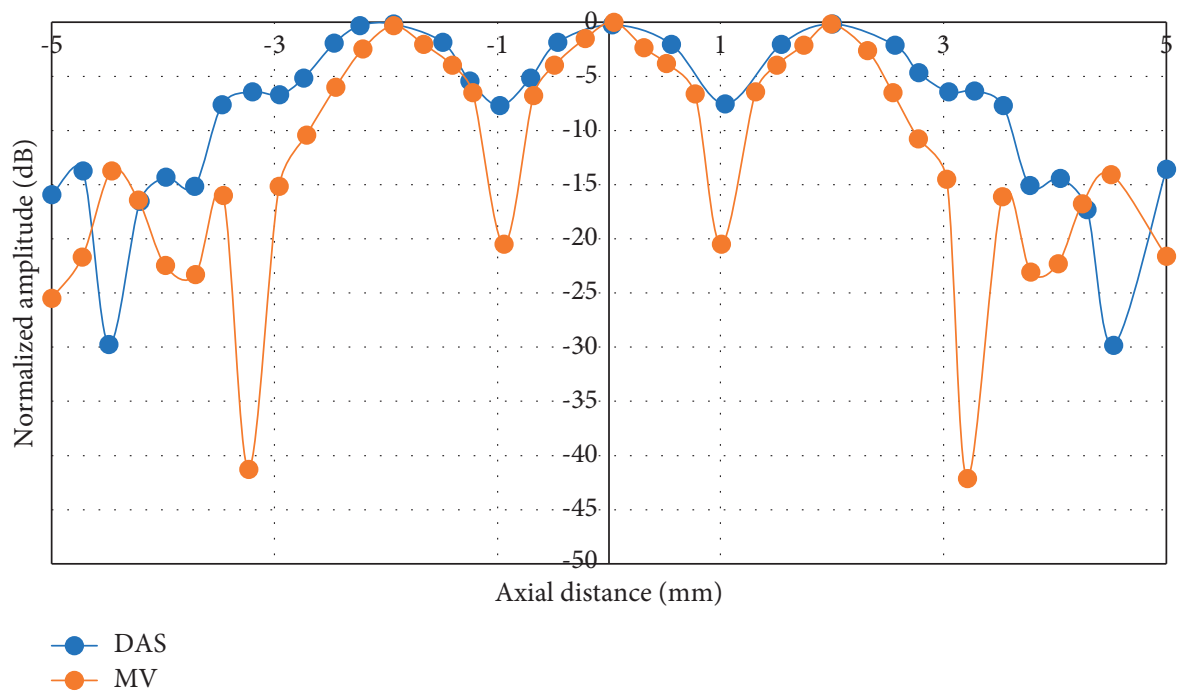

FIgURE 2: Comparison of imaging resolution at an axial distance of $50 \mathrm{~mm}$.

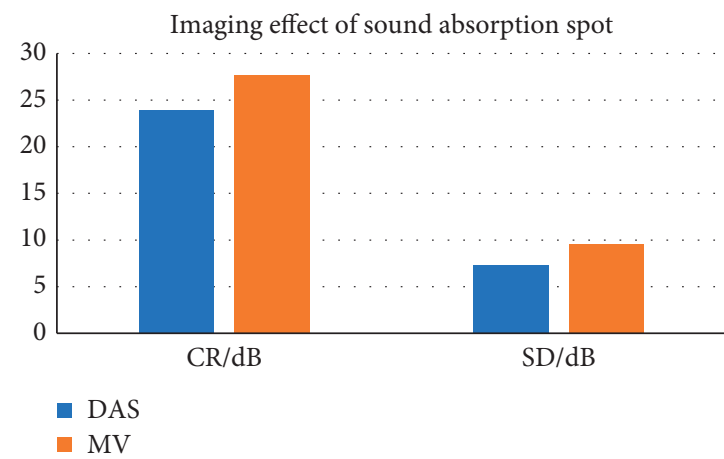

FIgURE 3: Contrast of sound absorption spot imaging.

TABle 1: General data of patients.

\begin{tabular}{lccc}
\hline Group & Number/cases & Age $/$ years & $\mathrm{BMI} / \mathrm{kg} / \mathrm{m}^{2}$ \\
\hline Experimental group & 119 & $34.33 \pm 7.34$ & $21.02 \pm 1.23$ \\
Control group & 94 & $35.34 \pm 7.67$ & $21.53 \pm 1.25$ \\
\hline
\end{tabular}

the patient's daily life or rest, analgesic drugs should be used to relieve the pain as directed by the doctor. This scale assessed the straightforwardness of image expression and had a high application value [14].

Each surgical patient compared the pain scale on the first, second, and third day after surgery to grade their own pain levels. Afterward, the pain degree of each group on the 1 st, 2 nd, and 3rd days after the operation was calculated according to the pain scores of the two groups of patients. The results are shown in Figure 7. From this data analysis, it was concluded that on the first postoperative day, the degree of neck or front chest wall pain in the experimental group was substantially less than that in the control group, with dramatical differences $(P<0.05)$. However, there was no considerable difference in pain between the two groups on the 2nd day and the 3rd day after the operation, and there was no statistical significance $(P=0.126$ and $P=0.483)$.
3.7. Comparison of Postoperative Recurrence and Metastasis between the Experimental Group and the Control Group. Postoperative follow-up indexes include Tg, thyroid, full set of thyroid function, and lymph node B ultrasound [15]. In the first year after the operation, it was reviewed once every 2 to 3 months. In the second year after the operation, it was reviewed once in 3 to 6 months. If there was no recurrence, it was reviewed once every 6 to 12 months. The average followup was $(15.1 \pm 7.5)$ months in the control group and $(15.5 \pm 7.7)$ months in the experimental group. During the follow-up, there was no obvious recurrence or metastasis in the two groups.

\section{Discussion}

Currently, considerable progress has been made in minimally invasive thyroid surgery [16, 17]. At present, the surgical indications of ultrasound-guided endoscopy in the treatment of papillary thyroid carcinoma have not formed a unified standard at home and abroad. However, with the continuous development and progress of endoscopy technology, the types of indications will gradually increase, and the previous contraindications will also decrease [18]. 
Postoperative routine pathological results

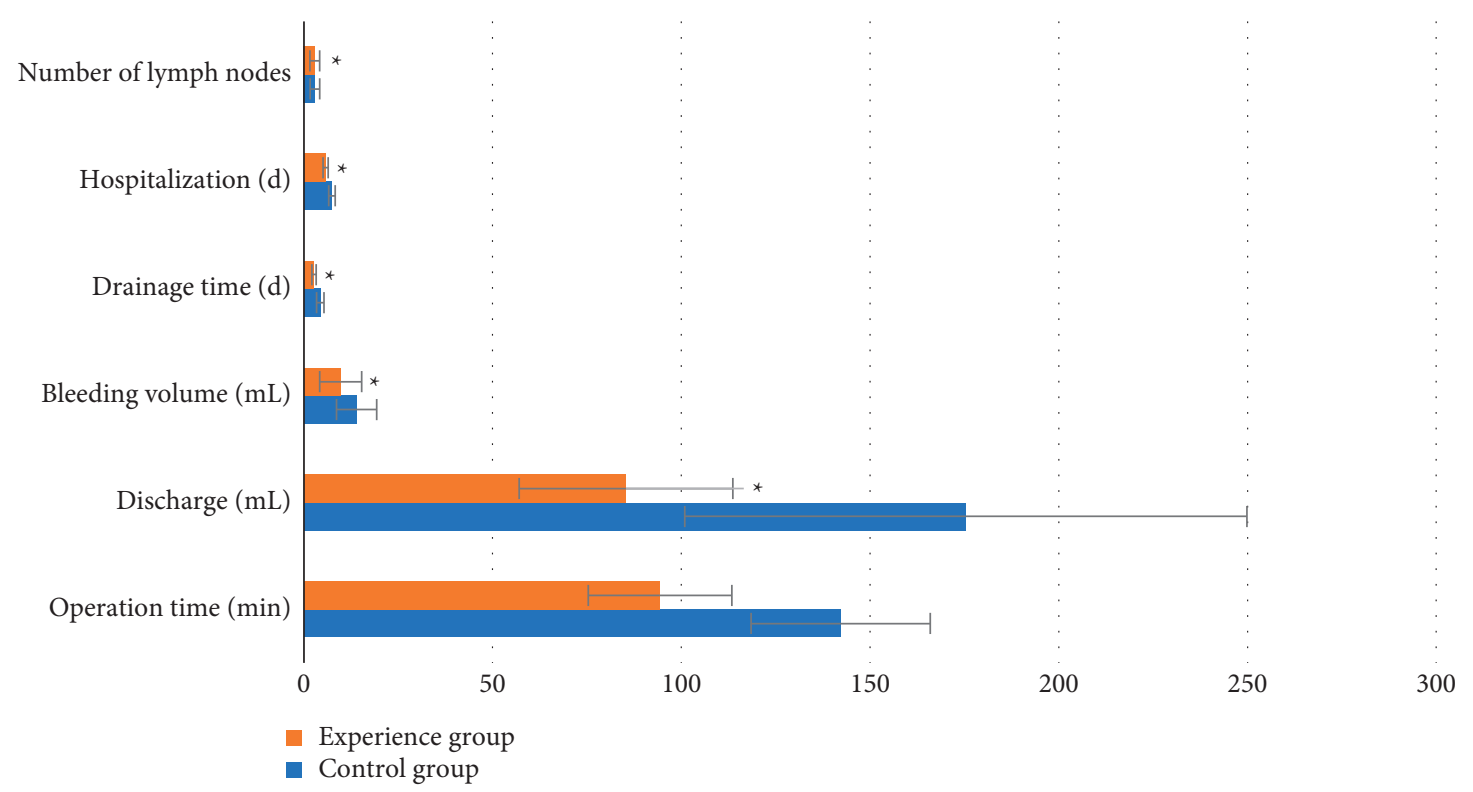

Figure 4: Postoperative routine pathological results ( ${ }^{*}$ compared with the control group, the difference was substantial $\left.(P<0.05)\right)$.

TABLE 2: Comparison of postoperative complications.

\begin{tabular}{lcccc}
\hline Group & $\begin{array}{c}\text { Skin } \\
\text { abnormalities/cases }\end{array}$ & $\begin{array}{c}\text { Subcutaneous congestion hematoma/ } \\
\text { cases }\end{array}$ & $\begin{array}{c}\text { Low calcium seizures/ } \\
\text { cases }\end{array}$ & $\begin{array}{c}\text { Postoperative bleeding/ } \\
\text { cases }\end{array}$ \\
\hline Control group & 5 & 4 & 3 & 1 \\
Experimental group & 3 & 5 & 2 & 0 \\
Statistics & 0.956 & 1.361 & 1.052 & 0.937 \\
$P$ & 0.872 & 0.467 & 1 & 1 \\
\hline
\end{tabular}

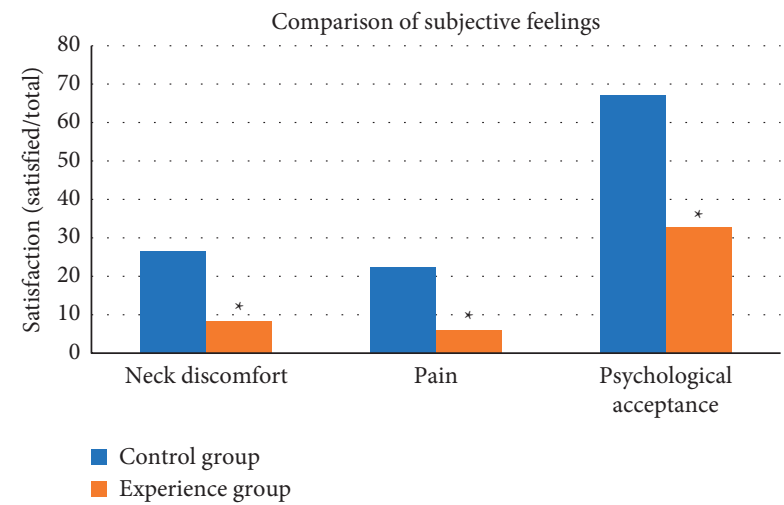

Figure 5: Subjective feelings of patients $\left({ }^{*}\right.$ compared with the control group, the difference was substantial $(P<0.05))$.

In this experiment, patients were randomly assigned to either conventional ultrasound-guided thoracoscopic surgery or ultrasound-guided thoracoscopic surgery based on artificial intelligence algorithms. In the analysis of postoperative results, compared with the experimental group, the average duration of surgery in the control group was substantially increased, with statistical significance $(P<0.05)$. The experimental group had less intraoperative blood loss than the control group, and the difference between the two groups was substantial
$(P<0.05)$. This proved that in the process of AI-based thoracoscopic surgery, the operator can quickly and accurately distinguish the arteries and veins in the operation area by using the identification function of ultrasound, which is safer and more reliable. Compared with the experimental group, the total amount of postoperative drainage was more in the control group, the duration of drainage tube placement was longer, and the length of hospital stay was longer. There were considerable differences between the two groups $(P<0.05)$. This indicated that the application of artificial intelligence algorithmbased ultrasound-guided endoscopic thyroid surgery was of high efficacy. It was also concluded that the incidence of complications in the experimental group was $13.83 \%$ and that in the control group was $14.29 \%$. There were little difference in the incidence of complications between the experimental group and the control group and no considerable difference in the incidence of postoperative complications between the two groups. These results indicated that ultrasound-guided endoscopic thyroid surgery based on an artificial intelligence algorithm had the same surgical safety as traditional surgery and may replace traditional surgery in a safe and feasible way [19].

Visual analog scoring was also used to compare anterior chest wall and neck pain in the two groups at $12 \mathrm{~h}, 24 \mathrm{~h}$, and $48 \mathrm{~h}$ after surgery. The results showed that there was a 


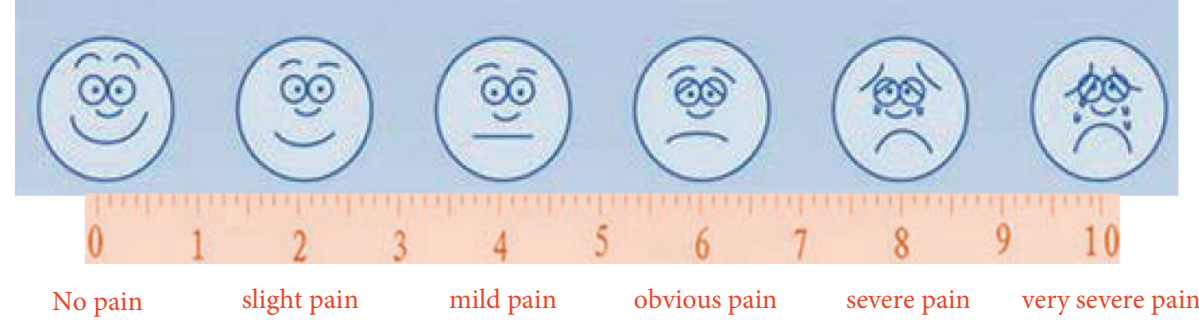

Figure 6: Facial expression pain scale.

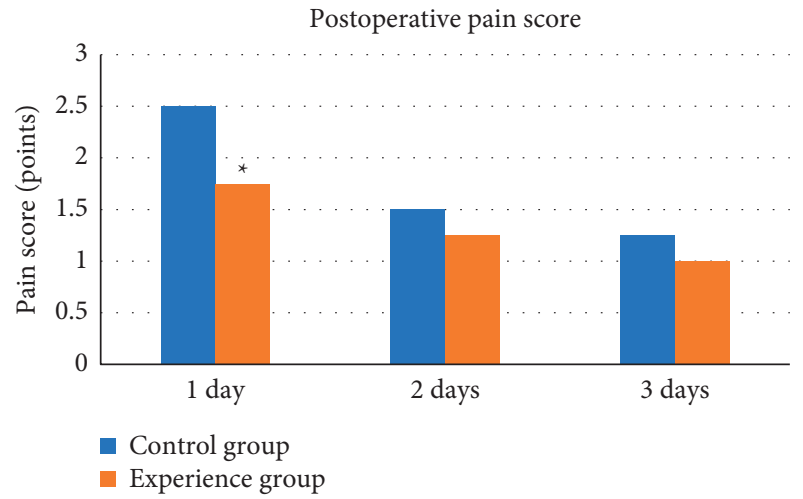

Figure 7: Comparison of the postoperative neck and anterior chest wall pain between the experimental group and the control group ( ${ }^{*}$ statistically significant difference compared with the control group $(P<0.05))$.

statistically remarkable difference in pain between the two groups on the first postoperative day $(P<0.05)$, while there was no considerable difference in the neck or anterior chest wall pain between the two groups on the second to third postoperative day $(P>0.05)$. This was enough to indicate that the postoperative pain sensation of endoscopic thyroid surgery based on an artificial intelligence algorithm was weaker than that of traditional surgery, and the rapid relief of patients' pain sensation was helpful for patients to step into normal life and work as soon as possible after surgery. However, studies pointed out that visual analog scoring is influenced by the subjective judgment of patients [20], and the accuracy of data conclusions needed to be further studied. The patients were followed up for six months after surgery, and the results of the postoperative cosmetic effect satisfaction survey showed that compared with the control group, patients in the experimental group were dramatically more satisfied with the cosmetic effect of the wound after surgery, and the difference between the two groups was statistically remarkable $(P<0.05)$. During the postoperative follow-up of the experimental group and the control group, the average follow-up was $(15.1 \pm 7.5)$ months in the control group and $(15.5 \pm 7.7)$ months in the experimental group. No tumor recurrence or metastasis was found. It indicated that endoscopic thyroid surgery based on an artificial intelligence algorithm also had the same high safety as traditional surgery in the short follow-up process, which can ensure the complete removal of thyroid tumor without tumor residue. However, long-term trials and more data were needed to confirm its long-term efficacy in treating thyroid papilloma.

\section{Conclusion}

Ultrasound imaging based on an artificial intelligence algorithm had a good effect on the treatment of papillary thyroid carcinoma surgery by thoracoscopic surgery. $\mathrm{Pa}$ tients' postoperative pain was weak, the aesthetic effect was good, and the psychological minimally invasive effect was substantial. Ultrasound imaging based on an artificial intelligence algorithm was beneficial to maintain patients' privacy and dramatically improved the surgical outcome and quality of life. It is a surgical method with high safety and good therapeutic effect that papillary thyroid carcinoma patients can trust. However, there are many postoperative drainages, and for large tumors and cases with lymph node metastasis, the length of the hospital stays increases, and the difficulty of surgery increases. Therefore, the treatment of patients with severe disease or with extensive lymph node metastasis should be carefully considered.

\section{Data Availability}

The data used to support the findings of this study are available from the corresponding author upon request.

\section{Conflicts of Interest}

The authors declare no conflicts of interest.

\section{References}

[1] C. Durante, G. Grani, L. Lamartina, S. Filetti, S. J. Mandel, and D. S. Cooper, "The diagnosis and management of thyroid nodules," JAMA, vol. 319, no. 9, pp. 914-924, 2018, Erratum in: JAMA. 2018 Apr 17;319(15):1622. PMID: 29509871.

[2] A. Prete, P. Borges de Souza, S. Censi, M. Muzza, N. Nucci, and M. Sponziello, "Update on fundamental mechanisms of thyroid cancer," Frontiers in Endocrinology, vol. 11, p. 102, 2020 PMID: 322316 39; PMCID: PMC7082927.

[3] U. C. Megwalu, "Observation versus thyroidectomy for papillary thyroid microcarcinoma in the elderly," Journal of Laryngology \& Otology, vol. 131, no. 2, pp. 173-176, 2017, Epub 2016 Dec 23. PMID: 28007045.

[4] K. M. Jayaprakasan and K. Ojha, "Ultrasound guidance in reproductive surgery," Best Practice \& Research Clinical Obstetrics \& Gynaecology, vol. 59, pp. 12-24, 2019, Epub 2019 May 30. PMID: 31279764.

[5] Z. Yu, S. U. Amin, M. Alhussein, and Z. Lv, "Research on disease prediction based on improved DeepFM and IoMT," IEEE Access, vol. 9, pp. 39043-39054, 2021. 
[6] Q. Huang, F. Zhang, and X. Li, "Machine learning in ultrasound computer-aided diagnostic systems: a survey," BioMed Research International, vol. 2018, pp. 1-10, 2018, PMID: 29687000; PMCID: PMC5857346.

[7] H. Hasegawa, "Apodized adaptive beamformer," Journal of Medical Ultrasonics, vol. 44, no. 2, pp. 155-165, 2017, Epub 2017 Jan 13. PMID: 28084559.

[8] N. Q. Nguyen and R. W. Prager, "Minimum variance approaches to ultrasound pixel-based beamforming," IEEE Transactions on Medical Imaging, vol. 36, no. 2, pp. 374-384, 2017, Epub 2016 Sep 15. PMID: 27654321.

[9] A. C. Luchies and B. C. Byram, "Deep neural networks for ultrasound beamforming," IEEE Transactions on Medical Imaging, vol. 37, no. 9, pp. 2010-2021, 2018, Epub 2018 Feb 26. PMID: 29994441; PMCID: PMC6109603.

[10] S. N. Christiansen, M. Østergaard, and L. Terslev, "Ultrasonography in gout: utility in diagnosis and monitoring," Clinical \& Experimental Rheumatology, vol. 36 Suppl 114, no. 5, pp. 61-67, 2018, Epub 2018 Oct 1. PMID: 30296983.

[11] J. Schnitzbauer, M. T. Strauss, T. Schlichthaerle, F. Schueder, and R. Jungmann, "Super-resolution microscopy with DNAPAINT," Nature Protocols, vol. 12, no. 6, pp. 1198-1228, 2017, Epub 2017 May 18. PMID: 28518172.

[12] L. J. Nowak and W. Steenbergen, "Reflection-mode acoustooptic imaging using plane wave ultrasound pulses," Journal of Biomedical Optics, vol. 26, no. 09, PMID: 34472243; PMCID: PMC8408765, Article ID 096001, 2021.

[13] Y. G. Pavlov and B. Kotchoubey, "EEG correlates of working memory performance in females," BMC Neuroscience, vol. 18, no. 1, p. 26, 2017 PMID: 28193 169; PMCID: PMC5307759.

[14] M. Kunz, S. F. Bunk, A. J. Karmann, K.-J. Bär, and S. Lautenbacher, "Conditioned pain modulation (CPM) effects captured in facial expressions," Journal of Pain Research, vol. Volume 14, pp. 793-803, 2021, PMID: 33790641 ; PMCID: PMC8001584.

[15] Y. Y. He, B. Zhang, Y. B. Zhang, Z. G. Xu, and P. Z. Tang, "[Clinical features of postoperative cervical lymph nodes recurrence in papillary thyroid carcinoma]," Zhonghua Er Bi Yan Hou Tou Jing Wai Ke Za Zhi, vol. 52, no. 6, pp. 416-420, 2017, Chinese PMID: 28635212.

[16] K. Tae, Y. B. Ji, C. M. Song, and J. Ryu, "Robotic and endoscopic thyroid surgery: evolution and advances," Clinical and Experimental Otorhinolaryngology, vol. 12, no. 1, pp. 1-11, 2019, Epub 2018 Sep 11. PMID: 3019668 8; PMCID: PMC6315214.

[17] M. Imran, Z. Mehmood, M. N. Baloch, and S. Altaf, "Endoscopic thyroid lobectomy vs. Conventional open thyroid lobectomy," Pakistan Journal of Medical Sciences, vol. 36, no. 4, pp. 831-835, 2020, PMID: 3249428 3; PMCID: PMC7260891.

[18] P. Miccoli and S. Bakkar, "Surgical management of papillary thyroid carcinoma: an overview," Updates in Surgery, vol. 69, no. 2, pp. 145-150, 2017, Epub 2017 Apr 12. PMID: 28405952.

[19] D. T. Nguyen, J. K. Kang, T. D. Pham, G. Batchuluun, and K. R. Park, "Ultrasound image-based diagnosis of malignant thyroid nodule using artificial intelligence," Sensors, vol. 20, no. 7, p. 1822, 2020 PMID: 32218230; PMCID: PMC7180806.

[20] A. P. Batarse, J. D. English, G. N. Frey, J. M. Piazza, and S. Akyalcin, "Referral patterns of pediatric dentists and general practitioners to orthodontists based on case complexity," American Journal of Orthodontics and Dentofacial Orthopedics, vol. 156, no. 1, pp. 61-66, 2019, PMID : 31256840. 